\title{
Patching and local-global principles for homogeneous spaces over function fields of $p$-adic curves
}

\author{
Jean-Louis Colliot-Thélène, Raman Parimala and Venapally Suresh
}

\begin{abstract}
Let $F=K(X)$ be the function field of a smooth projective curve over a $p$-adic field $K$. To each rank one discrete valuation of $F$ one may associate the completion $F_{v}$. Given an $F$-variety $Y$ which is a homogeneous space of a connected reductive group $G$ over $F$, one may wonder whether the existence of $F_{v}$-points on $Y$ for each $v$ is enough to ensure that $Y$ has an $F$-point. In this paper we prove such a result in two cases:

(i) $Y$ is a smooth projective quadric and $p$ is odd.

(ii) The group $G$ is the extension of a reductive group over the ring of integers of $K$, and $Y$ is a principal homogeneous space of $G$.

An essential use is made of recent patching results of Harbater, Hartmann and Krashen. There is a connection to injectivity properties of the Rost invariant and a result of Kato.
\end{abstract}

Mathematics Subject Classification (2010). 11G99, 14G99, 14G05, 11E72, 11E12, $20 \mathrm{G} 35$.

Keywords. Local-global principle, curves over local fields, homogeneous spaces.

\section{Introduction}

Let $K$ be a $p$-adic field, by which we mean a finite extension of a field $\mathbb{Q}_{p}$. Let $A$ be its ring of integers. Let $X / K$ be a smooth, projective, geometrically integral curve. Let $F=K(X)$ be the function field of $X$. This is a field of cohomological dimension 3. Let $\Omega$ denote the set of discrete valuations (of rank one) on the field $F$. Given $v \in \Omega$ we let $F_{v}$ denote the completion of $F$ at $v$.

We wonder whether in this context there is a local-global principle for the existence of rational points on homogeneous spaces of connected linear algebraic groups over $F$.

Conjecture 1. Let $F=K(X)$ be as above. Let $Y / F$ be a projective homogeneous space of a connected linear algebraic group. If $Y$ has points in all completions $F_{v}$, then it has an $F$-rational point.

Conjecture 2. Let $F=K(X)$ be as above. Let $G / F$ be a semisimple, simply connected group. If a class $\xi$ in the Galois cohomology set $H^{1}(F, G)$ has trivial image in each $H^{1}\left(F_{v}, G\right)$, then $\xi$ is trivial. In other words, if a principal homogeneous space under $G$ has points in all completions $F_{v}$, then it has an $F$-rational point. 
It is unlikely that Conjecture 2 holds for an arbitrary connected reductive group $G$, for instance for a torus. It definitely fails for $G$ a finite constant group, see $\S 6$.

As we explain in Section 5 (Theorem 5.4), Conjecture 2 may be proved for most quasisplit simply connected groups by using a combination of properties of the Rost invariant and a result of Kato [20].

In their recent paper [15], Harbater, Hartmann and Krashen have developed the patching technique of [14] to the point where they get local-global theorems for homogeneous spaces. Their main local-global theorems refer to some other families of overfields of $F$ than the family $\left\{F_{v}\right\}$ we consider here. But they manage to apply the technique to the extent that they give a radically new proof of the theorem by Parimala and Suresh [31] that any quadratic form in at least 9 variables over $K(X)$ ( $K$ as above, nondyadic, $X$ a curve over $K$ ) has a nontrivial zero.

Their techniques apply more generally to complete discrete valuation rings with arbitrary residue field.

In the present paper, we use the method and theorems of Harbater, Hartmann and Krashen to prove the following results.

1) For smooth quadrics of dimension at least 1 , which are projective homogeneous spaces under the associated special orthogonal group, under the assumption that the characteristic of the residue field of $K$ is not 2, we prove Conjecture 1. We actually prove the more general result (Theorem 3.1):

Let $A$ be a complete discrete valuation ring with fraction field $K$ and residue field $k$ of characteristic different from 2. Let $X$ be a smooth, projective, geometrically integral curve over $K$. Let $F=K(X)$ be the function field of $X$. Let $q$ be a nondegenerate quadratic form over $F$ in at least 3 variables. If for each discrete valuation of $F$, the form $q$ is isotropic over the completion of $F$ with respect to this valuation, then $q$ is isotropic over $F$.

2) We show (Theorem 4.8) that the statement of Conjecture 2 holds for any (fibrewise connected) reductive $A$-group $G$.

This relies on the following general result (Theorem 4.3):

Let $A$ be a complete discrete valuation ring, $K$ its field of fractions and $k$ its residue field. Let $\mathcal{X} / A$ be a projective, flat curve over Spec A. Assume that $\mathcal{X}$ is connected and regular. Let $F$ be the function field of $X$. Let $\Omega$ be the set of all discrete valuations on $F$. Let $G / A$ be a (fibrewise connected) reductive group. If there exists a connected linear algebraic group $H / F$ such that the $F$-group $\left(G \times{ }_{A} F\right) \times_{F} H$ is an $F$-rational variety, then the restriction map with respect to completions $H^{1}(F, G) \rightarrow \prod_{v \in \Omega} H^{1}\left(F_{v}, G\right)$ has a trivial kernel.

As mentioned above, an independent argument, which builds upon injectivity properties of the Rost invariant (which themselves rely on a case by case proof) and upon a theorem of Kato, yields a proof of Conjecture 2 for quasisplit, absolutely simple, simply connected groups over $F$ with no $E_{8}$-factor. 
In the final Theorem 5.5, we revert the process: we use Theorem 4.8 together with Bruhat-Tits theory to discuss the triviality of the kernel of the Rost invariant for split simply connected groups over a function field in one variable over a $p$-adic field. The result is classificationfree; in particular, it applies to $E_{8}$.

Throughout this paper, when we write "discrete valuation ring", we mean "discrete valuation ring of rank one", and when we write discrete valuation we mean valuation with value group $\mathbb{Z}$.

\section{Why the $u$-invariant should behave well for function fields over the $p$-adics}

The $u$-invariant of a field is the maximal dimension of anisotropic quadratic forms over that field. Let us start with some reminders from the paper [21] by Kato and Kuzumaki.

Let $r \geq 1$ be an integer. We say that a field $F$ is a $C_{r}^{0}$ field if the following condition holds:

For any finite field extension $F^{\prime}$ of $F$ and any integers $d \geq 1$ and $n>d^{r}$, for any homogeneous form over $F^{\prime}$ of degree $d$ in $n$ variables, the g.c.d. of the degrees of finite field extensions $F^{\prime \prime} / F^{\prime}$ over which the form acquires a nontrivial zero is 1 .

The condition amounts to requiring that the $F^{\prime}$-hypersurface defined by the form contain a zero-cycle of degree 1 over $F^{\prime}$.

Assume $\operatorname{char}(F)=0$. For each prime $l$, let $F_{l}$ be the fixed field of a pro- $l$-Sylow subgroup of the absolute Galois group of $F$. Any finite subextension of $F_{l} / F$ is of degree prime to $l$.

The field $F$ is $C_{r}^{0}$ if and only if each of the fields $F_{l}$ is $C_{r}$ in the usual sense (see [21, Lemma 1]). A finite field extension of a $C_{r}^{0}$-field is $C_{r}^{0}$. The following easy lemma does not appear in [21].

Lemma 2.1. Let $F$ be a field of characteristic zero. If $F$ is $C_{r}^{0}$ then a function field $E=F(X)$ in s variables over $F$ is $C_{r+s}^{0}$.

Proof. Let $E^{\prime}$ be a finite field extension of $E$. After replacing $F$ by a finite extension, which by assumption is still $C_{r}^{0}$, we may assume that $E^{\prime}$ is the function field $F(X)$ of a geometrically integral $F$-variety $X$. The field $F_{l}$ is $C_{r}$, hence by the classical transitivity properties (Lang, Nagata), the field $F_{l}(X)$, function field of $X \times{ }_{F} F_{l}$, is a $C_{r+s}$-field. Thus any form of degree $d$ over $F(X)$ in $n>d^{r+s}$ variables has nontrivial solutions in $F_{l}(X)$, hence in a finite extension of $F(X)$ of degree prime to $l$. As this applies to each prime $l$, this concludes the proof.

It is an open question whether $p$-adic fields have the $C_{2}^{0}$-property. An equicharacteristic zero analogue of that statement is proven in [6]. 
Proposition 2.2. Assume that $p$-adic fields have the $C_{2}^{0}$-property. Then over any function field $K(X)$ of transcendence degree $r$ over a $p$-adic field $K$, any quadratic form in strictly more than $2^{2+r}$ variables has a nontrivial zero.

Proof. By the previous lemma, such a quadratic form has a nontrivial zero in an extension of odd degree of the field $K(X)$. By a theorem of Springer [23, VII, Theorem 2.3] this implies that the quadratic form has a nontrivial zero in $K(X)$.

\section{A local-global principle for isotropy of quadratic forms}

Theorem 3.1. Let $A$ be a complete discrete valuation ring with fraction field $K$ and residue field $k$ of characteristic different from 2 . Let $X$ be a smooth, projective, geometrically integral curve over $K$. Let $F=K(X)$ be the function field of $X$. Let $q$ be a nondegenerate quadratic form over $F$ in at least 3 variables. Iffor each discrete valuation of $F$, the form $q$ is isotropic over the completion of $F$ with respect to this valuation, then $q$ is isotropic over $F$.

Proof. Suppose we are given a diagonal quadratic form $q=\left\langle a_{1}, \ldots, a_{n}\right\rangle$ over $F=$ $K(X)$ which is isotropic over the field of fractions of the completion of any discrete valuation ring of $F$.

Let us recall basic notation from [14] and [15].

Let $t$ denote a uniformizing parameter for $A$.

One may choose a regular proper model $X / A$ of $X / K$ such that there exists a reduced divisor $D$ with strict normal crossings which contains both the support of the divisor of the $a_{i}$ 's and the components of the special fibre of $X / A$. Let $Y=\mathcal{X} \times_{A} k$ denote the special fibre.

For the generic point $x_{i}$ of an irreducible component $Y_{i}$ of $Y$, there is an affine Zariski neighbourhood $W_{i}$ of $x_{i}$ in $\mathcal{X}$ such that the restriction of $Y_{i}$ to $W_{i}$ is a principal divisor.

Let $S_{0}$ be a finite set of closed points of the special fibre containing all singular points of $D$ and all points which lie on some $Y_{i}$ but not in the corresponding $W_{i}$.

Choose a finite $A$-morphism $f: \mathcal{X} \rightarrow \mathbb{P}_{A}^{1}$ as in [14, Proposition 6.6]. In particular, we have the following three properties. The set $S_{0}$ is contained in $S$, the inverse image under $f$ of the point at infinity of the special fibre $\mathbb{P}_{k}^{1}$. All the intersection points of two components $Y_{i}$ are in $S$. Each component $Y_{i}$ contains at least one point of $S$.

Let $U \subset Y$ run through the reduced, irreducible components of the complement of $S$ in $Y$. Each $U$ is a regular affine irreducible curve over $k$. Let $k[U]$ be the ring of regular functions on this curve. This is a Dedekind domain. We thus have $U=\operatorname{Spec} k[U]$. Let $k(U)$ denote the fraction field of $k[U]$. 
Since $\mathcal{X}$ is separated over $\operatorname{Spec} A$, the intersection of the affine open set $W_{i}$ with the affine open set $f^{-1}\left(\mathbf{A}_{A}^{1}\right)$ in $\mathcal{X}$ is an affine scheme Spec $B \subset \mathcal{X}$. The ring $B$ is an integral, noetherian, regular ring of dimension 2. There exists $s \in B$ such that $U=\operatorname{Spec}(B / s)$ and $t=u \cdot s^{r} \in B$ for some integer $r \geq 1$ and $u$ a unit in $B^{\times}$.

Let $C$ be the localisation of $B$ with respect to the multiplicative system $S$ of elements of $B$ which do not vanish at any point of $U$. We obviously have $C \subset B$. Let us show $C=R_{U}$. Since every point of $U$ is in $\operatorname{Spec} B$, the local ring $O_{X, x}$ of a point $x$ in $U$ is the local ring $B_{x}$, the localisation of $B$ at the point $x$. Thus $R_{U}$ which is the intersection of the rings $O_{X, x}$ as $x$ varies in $U$ is also the intersection of the localisations of $B$ at maximal ideals defining the points of $U$. If $a$ is an element of $R_{U}$, there exist finitely many points $x_{i}$ in $U$ and open neighbourhoods $V_{i}$ of $x_{i}$ in $U$, the $V_{i}$ covering $U$, and functions $g_{i}$ in $B$ nonvanishing on $V_{i}$ such that $a=f_{i} / g_{i}$, $f_{i} \in B$. Going modulo $s$, one concludes that there exist functions $h_{i} \in B$ such that $g=\sum_{i} h_{i} \cdot g_{i}$ is one modulo $s$, hence does not vanish on $U$. Then $a=\left(\sum_{i} h_{i} . f_{i}\right) / g$, hence $a$ belongs to $C$.

Since $R_{U}=C$ is a localisation of $B$, it is a regular, noetherian, regular ring. The inclusion $B \subset C$ induces an isomorphism $B / s \simeq C / s$.

Let $m$ be a maximal ideal in $C$. Assume $s \notin m$. Then $m+C . s=C$. Thus there exists $c \in C$ and $d \in m$ with $1=d+c . s$. Then $d=1-c . s$ does not vanish on $U$. Write $d=f / g$ with $f, g \in B$ and $g$ invertible on $U$. Then $f$ does not vanish on $U$, hence $d$ is invertible in $C$, which is a contradiction. Thus $s$ and $t=u \cdot s^{r}$ belong to each maximal ideal of $C=R_{U}$. By EGA IV, 7.8.3, one concludes that the $t$-adic completion $\hat{R}_{U}$, which is also the $s$-adic completion of $R_{U}$, is a regular domain ([15], Notation 3.3).

By definition, $F_{U}$ is the field of fractions of $\hat{R}_{U}$. We have $k[U]=R_{U} / s=\widehat{R}_{U} / s$.

For $P \in S$, the completion $\hat{R}_{P}$ of the local ring $R_{P}$ of $\mathcal{X}$ at $P$ is a domain ([15], Notation 3.3). By definition, the field $F_{P}$ is the field of fractions of $\hat{R}_{P}$.

For $p=(U, P)$ a pair with $P \in S$ in the closure of an irreducible component $U$ of the complement of $S$ in $Y$, one lets $R_{p}$ be the complete discrete valuation ring which is the completion of the localisation of $\hat{R}_{P}$ at the height one prime ideal corresponding to $U$. By definition, the field $F_{p}$ is the field of fractions of $R_{p}$.

By [14, Proposition 6.3], the field $F$ is the inverse limit of the finite inverse system of fields $\left\{F_{U}, F_{P}, F_{p}\right\}$.

Let us show that $q$ is isotropic over each field $F_{U}$.

Each diagonal entry $a_{i}$ of the form $q$ is supported only along $U$ in Spec $R_{U}$, thus is of the form $u . s^{j}$ where $u$ is a unit in $R_{U}$. Hence the quadratic form $q$ over $F$ is isomorphic to a quadratic form over $F$ of the shape

$$
\left\langle b_{1}, \ldots, b_{\rho}, s . c_{1}, \ldots, s . c_{\sigma}\right\rangle
$$

where $b_{i}$ and $c_{j}$ are units in $R_{U}$.

By hypothesis, $q$ is isotropic over the field of fractions of the completed local ring of $X$ at the generic point of $U$. By a theorem of Springer [23, VI, Proposition 1.9 (2)], 
this implies that the image of at least one of the two forms $q_{1}=\left\langle b_{1}, \ldots, b_{\rho}\right\rangle$ or $q_{2}=\left\langle c_{1}, \ldots, c_{\sigma}\right\rangle$ under the composite homomorphism $R_{U} \rightarrow k[U] \hookrightarrow k(U)$ is isotropic over $k(U)$.

Since the residue characteristic is not 2, each of the forms $q_{1}$ and $q_{2}$ defines a smooth quadric over $R_{U}$. In particular each of them defines a smooth quadric over $k[U]$. Since $k[U]$ is a Dedekind domain, if such a projective quadric has a point over $k(U)$, it has a point over $k[U]$. Since the quadric is smooth over $R_{U}$, a $k[U]$-point lifts to an $\widehat{R}_{U}$-point (compare the discussion after [15, Lemma 4.5]). Thus $q$ has a nontrivial zero over $F_{U}$.

Let us show that $q$ is isotropic over each field $F_{P}$. Let $P \in S$. The local ring $R_{P}$ of $\mathcal{X}$ at $P$ is regular. Its maximal ideal is generated by two elements $(x, y)$ with the property that any $a_{i}$ is the product of a unit, a power of $x$ and a power of $y$. Thus over the fraction field $F$ of $R_{P}$, the form $q$ is isomorphic to a form

$$
q_{1} \perp x . q_{2} \perp y . q_{3} \perp x y . q_{4}
$$

where each $q_{i}$ is a nondegenerate diagonal quadratic form over $R_{P}$. Let $R_{y}$ be the localization of $R_{P}$ at the prime ideal $(y)$. This is a discrete valuation ring with fraction field $F$ and with residue field $E$ the field of fractions of the discrete valuation ring $R_{P} /(y)$. By hypothesis, the form $\left(q_{1} \perp x . q_{2}\right) \perp y .\left(q_{3} \perp x q_{4}\right)$ is isotropic over the field of fractions of the completion of $R_{y}$. By Springer's theorem [23, VI, Proposition 1.9(2)], this implies that over $E$ the reduction of either $\left(q_{1} \perp x . q_{2}\right)$ or $\left(q_{3} \perp x q_{4}\right)$ is isotropic. Since $x$ is a uniformizing parameter for $R_{P} /(y)$, by Springer's theorem [23, VI, Proposition 1.9 (2)], this last statement implies that over the residue field of $R_{P} /(y)$, the reduction of one of the forms $q_{1}, q_{2}, q_{3}, q_{4}$ is isotropic. But then one of these forms is isotropic over $\widehat{R}_{P}$, hence over the field $F_{P}$ which is the fraction field of $\hat{R}_{P}$.

The quadric $Z / F$ defined by the vanishing of $q$ is a homogeneous space of the group $\mathrm{SO}(q)$ over $F$, which since $q$ is of rank at least 3 is a connected group. By Witt's result, for any field $L$ containing $F$, the group $\mathrm{SO}(q)(L)$ acts transitively on $Z(L)$. The $F$-variety underlying $\mathrm{SO}(q)$ is $F$-rational (Cayley parametrization). We have $Z\left(F_{U}\right) \neq \emptyset$ for each $U$ and $Z\left(F_{P}\right) \neq \emptyset$ for each $P \in S$. By [15, Theorem 3.7], we get $Z(F) \neq \varnothing$.

Remark 3.2. Note that in the proof the only discrete valuation rings which are used are the local rings at a point of codimension 1 on a suitable regular proper model $\mathcal{X}$ of $X$. See however Remark 3.6.

Remark 3.3. The theorem does not extend to forms in 2 variables. See Remark 4.4 and Section 6.

The following corollary is a variant of a theorem of Harbater, Hartmann and Krashen [15, Theorem 4.10]. 
Corollary 3.4. Let $A$ be a complete discrete valuation ring with fraction field $K$ and residue field $k$ of characteristic different from 2 . Let $r \geq 1$ be an integer. Assume that any quadratic form in strictly more than $2 r$ variables over any function field in one variable over $k$ is isotropic.

Then any quadratic form in strictly more than $4 r$ variables over the function field $F=K(X)$ of a curve $X / K$ is isotropic.

Proof. Let $L$ be a finite field extension of $K$. This is a complete discrete value field with residue field a finite extension $l$ of $k$. The hypothesis made on quadratic forms over function fields in one variable over $k$, in particular quadratic forms over the field $l(t)$, and Springer's theorem ([23, VI, Corollary 1.10]) applied to the field $l((t))$ imply that any quadratic form in strictly more than $r$ variables over $l$ has a zero. Another application of Springer's theorem then implies that any quadratic form in strictly more than $2 r$ variables over $L$ is isotropic.

Let $q$ be a quadratic form in $n$ variables over $F$ with $n>4 r$. By Theorem 3.1 and Remark 3.2, to prove the corollary it suffices to show that $q$ is isotropic over $F_{v}$ for every discrete valuation $v$ with residue field either a function field in one variable over $k$ or a finite extension of $K$. By the hypothesis, the preceding paragraph and Springer's theorem, the quadratic form $q$ is isotropic over such an $F_{v}$.

Corollary 3.4 in its turn is a generalization of the main result of [31]:

Corollary 3.5. If $K$ is a nondyadic p-adic field, any quadratic form in at least 9 variables over a function field in one variable over $K$ has a nontrivial zero.

Remark 3.6. In Theorem 3.1, it is not enough to consider the discrete valuation rings corresponding to the codimension 1 points of a given regular proper model $\mathcal{X} / A$.

Let $p$ be an odd prime and $a$ a unit in $\mathbb{Z}_{p}$ which is not a square. Rowen, Sivatski, Tignol [33, Corollary 5.3] (see also [19]) have shown that the tensor product $D=(a, p) \otimes(t, a(p-t))$ of quaternion algebras over $F=\mathbb{Q}_{p}(t)$ is a division algebra. The tensor product $(a, b) \otimes(c, d)$ of two quaternion algebras over a field $F(\operatorname{char} F \neq 2)$ is a division algebra, i.e. has index 4 , if and only if the associated Albert form $\langle-a,-b, a b, c, d,-c d\rangle$ is anisotropic over $F$. Thus the quadratic form $q=\langle-a,-p, a p, t, a(p-t),-a t(p-t)\rangle$ is a 6-dimensional anisotropic quadratic form over $F=\mathbb{Q}_{p}(t)$.

Let $\mathcal{X}=\mathbb{P}_{\mathbb{Z}_{p}}^{1}$ be the projective line over $\mathbb{Z}_{p}$. The codimension one points $v$ of $X$ are given by irreducible monic polynomials in $\mathbb{Q}_{p}[t]$, by $1 / t$ and by the height one prime ideal of $\mathbb{Z}_{p}[t]$ generated by $p$. Let $F=\mathbb{Q}_{p}(t)$, and let $F_{v}$ denote the completion of $F$ at a discrete valuation $v$ of $F$.

The residue field at a point $v$ of codimension 1 of $X$ is either a $p$-adic field or $\mathbb{F}_{p}(t)$. Any quadratic form in at least 5 variables over such a field is isotropic. At any prime $v$ of codimension 1 of $\mathcal{X}$ different from $p, t,(p-t), 1 / t$, the form $q$ therefore has a zero 
over $F_{v}$. At $v=t$, one of the residue forms is $\langle-a,-p, a p, a p\rangle$ which is isotropic over the residue field $\mathbb{Q}_{p}$, since $\langle-1, a, a\rangle$ is. At $v=t-p$, one of the residue forms of $q$ is $\langle-a,-p, a p, p\rangle$ and this form is clearly isotropic. At $v=(1 / t)$, one of the residue forms of $q$ is $\langle-a,-p$, ap, $a\rangle$ which is clearly isotropic. At $v=p$, one of the residue forms over the field $\mathbb{F}_{p}(t)$ is $\langle-a, t,-a t, a\rangle$ which again is clearly isotropic. Thus the quadratic form $q$ is isotropic over each field $F_{v}$ corresponding to a point of codimension 1 on $\mathcal{X}$.

Theorem 3.1 and the result of [33] show that there must exist another completion $F_{v}$, corresponding to a codimension 1 point on another model of $\mathbb{P}_{\mathbb{Z}_{p}}^{1}$, at which the form is anisotropic.

Note that on $\mathbb{P}_{\mathbb{Z}_{p}}^{1}$, the divisor associated to the quadratic form does not have normal crossings at the point defined by the ideal $(p, t) \subset \mathbb{Z}_{p}[t]$ (compare the proof of Theorem 3.1). It is thus natural to blow up the corresponding point. In practice, one introduces a new variable $x$ and one sets $t=p x$. The quadratic form $q$ now reads $\langle-a,-p, a p, p x, a p(1-x),-a x(1-x)\rangle$. At the prime ideal $p$ of $\mathbb{Z}_{p}[x]$, with residue field $\mathbb{F}_{p}(x)$, the two residue forms are $\langle-a,-a x(1-x)\rangle$ and $\langle-1, a, x, a(1-x)\rangle$. Since $x(x-1)$ is not a square in $\mathbb{F}_{p}(x)$, the first form is clearly anisotropic. As for the second one, the two residue forms of $\langle-1, a, x, a(1-x)\rangle$ at the valuation of $\mathbb{F}_{p}(x)$ with uniformizing parameter $1 / x$ are anisotropic over $\mathbb{F}_{p}$, because $a$ is not a square.

At any closed point of $\mathbb{P}_{\mathbb{Z}_{p}}^{1}$ different from the point defined by $(p, t) \subset \mathbb{Z}_{p}[t]$ the form $q$ admits a reduction of the shape $\langle a,-a\rangle$, hence it is isotropic over the fraction field of the complete local ring at such a point. The $\mathbb{Z}_{p}$-homomorphism $\mathbb{Z}_{p}[t] \rightarrow \mathbb{Z}_{p}[x]$ sending $t$ to $p x$ sends the ideal $(p, t)$ of $\mathbb{Z}_{p}[t]$ to the ideal $p$ of $\mathbb{Z}_{p}[x]$. It induces an injective homomorphism of the corresponding complete local rings, hence an embedding of their fraction fields. The above argument shows that $q$ is anisotropic over the bigger fraction field. It is thus anisotropic over the fraction field of the completion of $\mathbb{Z}_{p}[t]$ at the maximal ideal $(p, t)$.

Remark 3.7. The following question was raised by D. Harbater. Let $A, K, X$ and $F=K(X)$ be as in Theorem 3.1. Suppose a projective homogeneous variety $Z$ over $F$ under an $F$-rational connected linear algebraic group has points in the field of fractions of the completions at closed points of all possible regular proper models of $X$ over $A$. Does $Z$ admit a rational point over $F$ ? The following example gives a negative answer to this question, already with $Z$ a quadric.

Let $p$ be an odd prime, let $X / \mathbb{Z}_{p}$ be a smooth curve over $\mathbb{Z}_{p}$ of relative genus at least 1. Let $X / \mathbb{Q}_{p}$ be its generic fibre and let $Y / \mathbb{F}_{p}$ be its special fibre. There exist two quaternion division algebras $H_{1}$ and $H_{2}$ over the function field $\mathbb{F}_{p}(Y)$ whose ramification loci on $Y$ are disjoint. Let $\bar{q}_{1}$ and $\bar{q}_{2}$ be the reduced norms attached to these two quaternion division algebras. Let $q_{1}, q_{2}$ be lifts of these quadratic forms to the local ring $R$ of the generic point of $Y$ on $X$. Consider the quadratic form $q=q_{1} \perp p . q_{2}$ over the function field $F=\mathbb{Q}_{p}(X)$. This form is isotropic over the 
fraction field of the local ring of any closed point $P \in \mathcal{X}$. Indeed at any such point either the form $q_{1}$ or the form $q_{2}$ has good reduction.

On the other hand, the form $q$ is anisotropic over $F$ since both $q_{1}$ and $q_{2}$ are anisotropic over the $p$-adic completion of the local ring $R$, whose uniformizing parameter is $p$.

Since the genus of $X$ is at least 1 , by a well known result of Shafarevich [37] and Lichtenbaum [24], the curve $X / \mathbb{Z}_{p}$ is an absolute minimal model of $X$ over $\mathbb{Z}_{p}$. Thus if $\mathcal{X}^{\prime} / \mathbb{Z}_{p}$ is another model, there is a birational $\mathbb{Z}_{p}$-morphism $X^{\prime} \rightarrow \mathcal{X}$. The argument given above then shows that $q$ is isotropic on the fraction field of the local ring of any closed point of $\mathcal{X}^{\prime}$.

Remark 3.8. Let $F$ be a function field in one variable over a $p$-adic field $k$.

For quadratic forms in 3 or 4 variables, there is a refined local-global principle for isotropy of quadratic forms: one only needs to take into account discrete valuations which are trivial on $K$. The case of 3 variables is a consequence of a theorem of Lichtenbaum [25], based on Tate's duality theorem for abelian varieties over a $p$-adic field: for $X / K$ a smooth projective geometrically connected curve $X$ over a $p$-adic field $K$, if an element of the Brauer group of $X$ vanishes after evaluation at each closed point of $X$, then it is zero. The case of forms in 4 variables follows from the case of 3 variables by passing over to the discriminant extension of the 4-dimensional form. This should be compared with Theorem 3.1. As Remark 3.6 shows, such a refined local-global principle does not hold for forms in 6 variables. It actually does not hold for forms in 5, 6, 7 or 8 variables, as the following argument shows.

Let $K$ be a $p$-adic field. Suppose we are given a smooth complete intersection $Y$ of two quadrics given by a system of two quadratic forms $f=g=0$ in projective space $\mathbb{P}_{K}^{n}$ such that $Y(K)=\varnothing$. By a theorem due independently to Amer (unpublished) and to Brumer [3], the quadratic form $f+t g$ in $n+1$ variables over the field $K(t)$ then does not have a nontrivial zero. The hypothesis of smoothness of $Y$ ensures that over any completion $F_{v}$ of $F=K(t)$ at a place trivial on $K$, the form $f+t g$ contains a good reduction subform of rank at least $n$. Since $K$ is $p$-adic, for $n \geq 5$, such a form has a nontrivial zero. Hence for $n \geq 5$, that is from 6 variables onwards, the form $f+\operatorname{tg}$ over $F=K(t)$ has a nontrivial zero in each completion of $F$ at a place trivial on $K$. It remains to exhibit such systems of forms as above. By a classical compacity argument, to prove the existence of such a smooth $Y$, it is enough to produce an arbitrary complete intersection of two quadrics in $\mathbb{P}_{K}^{n}$ with no $K$-point. But that is easy. Let $f\left(x_{1}, x_{2}, x_{3}, x_{4}\right)$ be the norm form of the nontrivial quaternion algebra over $K$. Then the system $f\left(x_{1}, x_{2}, x_{3}, x_{4}\right)=0, f\left(x_{5}, x_{6}, x_{7}, x_{8}\right)=0$ defines such a complete intersection in $\mathbb{P}^{7}$, and one gets suitable systems in lower dimensional projective space by letting some variables vanish. With $f$ as above, $m$ a suitable integer, and $h_{1}$ and $h_{2}$ suitable diagonal quadratic forms, one can produce a smooth 
complete intersection without $K$-point of the shape:

$$
f\left(x_{1}, x_{2}, x_{3}, x_{4}\right)+p^{m} h_{1}\left(x_{5}, x_{6}, x_{7}, x_{8}\right)=0,
$$

and

$$
p^{m} h_{2}\left(x_{1}, x_{2}, x_{3}, x_{4}\right)+f\left(x_{5}, x_{6}, x_{7}, x_{8}\right)=0 .
$$

There also exist smooth intersections of two quadrics

$$
f\left(x_{1}, x_{2}, x_{3}, x_{4}, x_{5}\right)=g\left(x_{1}, x_{2}, x_{3}, x_{4}, x_{5}\right)=0
$$

in $\mathbb{P}_{K}^{4}$ such that at any completion $F_{v}$ of $F=K(t)$ at a place trivial on $K$ the form $f+t g$ has an $F_{v}$-point but has no $F$-point. Here is one example. Let $p$ be a prime, $p \equiv 1 \bmod 4$. Let $K=\mathbb{Q}_{p}, u \in \mathbb{Z}_{p}^{*}$ a unit which is not a square and $s \geq 2$ an integer. Then take

$$
f=x_{1}^{2}+u x_{2}^{2}+p x_{3}^{2}+u p^{2 s} x_{4}^{2}+p^{2 s-2} x_{5}^{2}
$$

and

$$
g=p^{4 s+1} x_{1}^{2}+p^{4 s} x_{2}^{2}+u p^{2 s} x_{3}^{2}+x_{4}^{2}+p x_{5}^{2} .
$$

One immediately checks that this defines a smooth complete intersection $Y$ in $\mathbb{P}_{\mathbb{Q}_{p}}^{4}$. The system $f=g=0$ has no primitive solution modulo $p^{2}$, hence $Y$ has no rational point over $\mathbb{Q}_{p}$. Let $v$ be a place of $F$ trivial on $K$. If $f+t g$ has good reduction at $v$, then its reduction has rank 5 over the residue field, hence is isotropic. The places $v$ at which $f+t g$ has bad reduction are 5 distinct rational points of $\operatorname{Spec} \mathbb{Q}_{p}[t]$. At each of these completions, $f+t g$ has a good reduction subform whose reduction is isotropic of rank 4 over $\mathbb{Q}_{p}$.

\section{A local-global principle for principal homogeneous spaces under certain linear algebraic groups}

Given a scheme $X$ and a smooth $X$-group scheme $G$, we let $H^{1}(X, G)$ denote the first Čech cohomology set for the étale topology on $X$.

The following lemma is known ([16, Lemma 4.1.3]).

Lemma 4.1. Let $A$ be a discrete valuation ring, $K$ its fraction field, $\hat{A}$ its completion and $\hat{K}$ the field of fractions of $\widehat{A}$. Let $G / A$ be a reductive group (with connected fibres). Then the fibre product of $H^{1}(K, G)$ and $H^{1}(\hat{A}, G)$ over $H^{1}(\widehat{K}, G)$ is $H^{1}(A, G)$.

Proof. Let $G \subset \mathrm{GL}_{n, A}$ be a closed embedding of $A$-groups and let $Z / A$ denote the quotient $\mathrm{GL}_{n, A} / G$ (see [8, Corollary 6.12]). For any local $A$-algebra $B$, by [13, III, 3.2.4 and 3.2.5] we have an exact sequence of pointed sets

$$
\mathrm{GL}_{n}(B) \rightarrow Z(B) \rightarrow H^{1}(B, G) \rightarrow 1 .
$$


More precisely, the natural map $Z(B) \rightarrow H^{1}(B, G)$ induces a bijection between the quotient of $Z(B)$ by the left action of $G L_{n}(B)$ and the set $H^{1}(B, G)$. That the natural map $H^{1}(A, G) \rightarrow H^{1}(K, G)$ is injective is a theorem of Nisnevich [29]: there it is proven that the kernel is trivial for any reductive $A$-group $G$, a known twisting argument (an étale cohomology variant of [35], §5.4, p. 47, or [22], 28.9, p. 367) then implies that the map is injective. Let $x \in Z(K)$ be a lift of $\xi \in H^{1}(K, G)$. If the image of $\xi$ in $H^{1}(\widehat{K}, G)$ comes from $H^{1}(\widehat{A}, G)$, then there exists $y \in Z(\widehat{A})$ and $\rho \in \mathrm{GL}_{n}(\hat{K})$ such that $\rho . x=y \in Z(\hat{K})$. The set $\mathrm{GL}_{n}(K)$ is dense in $\mathrm{GL}_{n}(\hat{K})$, the map $\mathrm{GL}_{n}(\hat{K}) \rightarrow Z(\widehat{K})$ is continuous, and $Z(\widehat{A})$ is open in $Z(\widehat{K})$. We may thus find $g \in \mathrm{GL}_{n}(K)$ close enough to $\rho$ that $g . x$ lies in $Z(\hat{A})$. Now $g \cdot x$ lies in $Z(K) \cap Z(\widehat{A})=Z(A)$.

Unramified classes. Given $G / A$ as above, and $\xi_{K} \in H^{1}(K, G)$, one says that $\xi_{K}$ is unramified at $A$ if it lies in the image of $H^{1}(A, G)$. By the above lemma, it then comes from a well defined element $\xi_{A} \in H^{1}(A, G)$. By the same lemma, the condition is equivalent to requiring that the image of $\xi_{K}$ in $H^{1}(\hat{K}, G)$ comes from a well defined element $\xi_{\hat{A}} \in H^{1}(\hat{A}, G)$. Let $k$ denote the residue class field of $A$ and $\widehat{A}$. If a class $\xi_{K} \in H^{1}(K, G)$ is unramified, one may then consider its evaluation $\xi_{k} \in H^{1}\left(k, G_{k}\right)$. It is given by the image of $\xi_{A}$, or of $\xi_{\widehat{A}}$, in $H^{1}\left(k, G_{k}\right)$.

Theorem 4.2. Let $A$ be a complete discrete valuation ring, $K$ its field of fractions and $k$ its residue field. Let $\mathcal{X} / A$ be a projective, flat curve over $\operatorname{Spec} A$. Assume that $\mathcal{X}$ is connected and regular. Let $F$ be the function field of $\mathcal{X}$. Let $G / A$ be a (fibrewise connected) reductive group. Let $\xi_{F} \in H^{1}(F, G)$ be a class which is unramified at all codimension 1 points of $\mathcal{X}$.

(i) There exists $\xi \in H^{1}(\mathcal{X}, G)$ whose image in $H^{1}(F, G)$ is $\xi_{F}$.

(ii) If moreover the (reduced) components of the special fibre are regular, and for any such component $Y$ the image $\xi_{k(Y)}$ in $H^{1}(k(Y), G)$ is trivial, then at any point $P$ of codimension 1 or 2 of $\mathcal{X}$, with residue field $\kappa(P)$, the image $\xi_{P} \in H^{1}(\kappa(P), G)$ is trivial.

(iii) If moreover there exists a connected linear algebraic group $H / F$ such that the $F$-group $\left(G \times_{A} F\right) \times_{F} H$ is an $F$-rational variety, then $\xi_{F}=1 \in H^{1}(F, G)$.

Proof. (i) By definition of an unramified class, for each point $P$ of codimension 1 on $\mathcal{X}$ there exists $\xi_{P} \in H^{1}\left(O_{X, P}, G\right)$ with image $\xi_{F}$ over the fraction field $F$ of $O_{X, P}$. By Nisnevich's theorem [29], the class $\xi_{P}$ is uniquely defined.

There then exists a Zariski open set $V \subset \mathcal{X}$ which contains all points of dimension 1 of $\mathcal{X}$ and an element of $H^{1}(V, G)$ with image $\xi$ in $H^{1}(F, G)$ (see the proof of Proposition 6.8 in [8]). Since $\mathcal{X}$ is regular and of dimension 2, [8, Theorem 6.13] shows that one may take $V=\mathcal{X}$. We thus have a class $\xi \in H^{1}(\mathcal{X}, G)$ with image $\xi_{F}$ in $H^{1}(F, G)$. In other terms, we have a torsor $E$ over $\mathcal{X}$ under the $\mathcal{X}$-group 
scheme $G_{X}=G \times_{A} \mathcal{X}$, whose restriction over the generic point of $\mathcal{X}$ has class $\xi_{F} \in H^{1}(F, G)$.

(ii) Let $P$ be a closed point of the special fibre. Let $k(P)$ denote the residue field at $P$. Let $Y$ be a component of the special fibre which contains $P$. Since we assumed the components to be regular, the local ring $O_{Y, P}$ is a discrete valuation ring. The image of the class of $\xi$ in $H^{1}\left(O_{Y, P}, G\right)$ is now trivial because its image in $H^{1}(k(Y), G)$ is trivial (easy case of [29]). Hence the image of $\xi$ in $H^{1}(k(P), G)$ is trivial.

Now let $P$ be a closed point of the generic fibre. Let $B$ denote the integral closure of $A \subset K$ in the residue field $K(P)$. This is a complete discrete valuation ring. There exists an $A$-morphism $\operatorname{Spec} B \rightarrow X$ which extends the inclusion of $P$ in $X$. The image of the special point of $\operatorname{Spec} B$ is a point in the special fibre of $X / A$, hence it lies on some component $Y$. By the above argument, the evaluation of $\xi$ at that point is trivial. Thus the inverse image of $\xi$ on Spec $B$ is a $G$-torsor with trivial special fibre. By Hensel's lemma on the complete local ring $B$, this inverse image is trivial. Hence $\xi_{P}$ is trivial at $P$, which is the generic point of Spec $B$.

(iii) The hypothesis in (ii) ensures that for each component $Y$ of the special fibre there exists a dense open set $U_{Y} \subset Y$ such that the restriction of $\xi$ to $U_{Y}$ is trivial.

We may assume that each $U_{Y}$ meets no component but $Y$. The complement of the union of $U_{Y}$ 's in the special fibre is a finite set $S$ of points.

By Proposition 6.6 of [14], there exists a finite $A$-morphism $f: \mathcal{X} \rightarrow \mathbb{P}_{A}^{1}$ with $S \subset f^{-1}\left(\infty_{k}\right)$. One now replaces the family of $U_{Y}$ 's by the family $U$ of irreducible components of $f^{-1}\left(\mathbb{A}_{k}^{1}\right)$. This replaces each $U_{Y}$ by a nonempty affine open set of $U_{Y}$ and one replaces $S$ by $f^{-1}\left(\infty_{k}\right)$.

By (ii), for each closed point $P$ of the special fibre, for $E$ as in (i) we have $E(k(P)) \neq \emptyset$. Since $E / \mathcal{X}$ is smooth, this implies $E\left(\widehat{R}_{P}\right) \neq \emptyset$, hence $E\left(F_{P}\right) \neq \emptyset$ (notation as in Theorem 3.1 and as in [14]).

For each open set $U=U_{Y}$, the restriction of $E$ over $U$ is a trivial $G_{U}$-torsor. Since $E / X$ is smooth, this implies $E\left(\widehat{R}_{U}\right) \neq \emptyset$ hence $E\left(F_{U}\right) \neq \varnothing$.

Since $E \times x F$ is a principal homogeneous space under the $F$-algebraic group $G \times{ }_{A} F$, for any field extension $L$ of $F$, the group $G(L)$ acts transitively on $E(L)$. An application of [15, Theorem 3.7 and Corollary 3.8] now yields $E(F) \neq \varnothing$, i.e. $\xi=1 \in H^{1}(F, G)$.

Theorem 4.3. Let $A$ be a complete discrete valuation ring, $K$ its field of fractions and $k$ its residue field. Let $\mathcal{X} / A$ be a projective, flat curve over $\operatorname{Spec} A$. Assume that $\mathcal{X}$ is connected and regular. Let $F$ be the function field of $\mathcal{X}$. Let $\Omega$ be the set of all discrete valuations on $F$.

(i) Let $G / A$ be a (fibrewise connected) reductive group. If there exists a connected linear algebraic group $H / F$ such that the $F$-group $\left(G \times_{A} F\right) \times_{F} H$ is an $F$-rational variety, then the restriction map with respect to completions $H^{1}(F, G) \rightarrow$ $\prod_{v \in \Omega} H^{1}\left(F_{v}, G\right)$ has a trivial kernel. 
(ii) The restriction map of Brauer groups $\mathrm{Br} F \rightarrow \prod_{v \in \Omega} \mathrm{Br} F_{v}$ has a trivial kernel.

Proof. Statement (i) immediately follows from the previous theorem. As for statement (ii), it follows from (i) applied to the projective linear groups $\mathrm{PGL}_{n}$.

Remark 4.4. Using totally split unramified coverings of models of Tate curves over a $p$-adic field (see [34]), one sees that Theorem 4.3 (i) does not in general hold for nonconnected groups, for example for $G=\mathbb{Z} / 2$. A concrete example is given by the elliptic curve $E$ with affine equation $y^{2}=x(1-x)(x-p)$ over the $p$-adic field $\mathbb{Q}_{p}$ ( $p$ odd). The rational function $1-x$ is not a square in the function field $F=\mathbb{Q}_{p}(E)$, but it becomes a square in each completion $F_{v}$ of $F$. This example is discussed in the appendix to this paper (Section 6). This implies that the patching results of [15] in general do not extend to nonconnected groups.

Lemma 4.5. Let $A$ be the ring of integers of a $p$-adic field $K$, let $k$ be its residue field. Let $G / A$ be a (fibrewise connected) reductive group. Then there exists a connected linear algebraic group $H / K$ such that the $K$-group $\left(G \times_{A} K\right) \times_{K} H$ is $K$-rational.

Proof. Let $Z / A$ be the $A$-scheme of Borel subgroups of $G$. This is a proper and smooth scheme over Spec $A$. The special fibre $G_{0}=G \times{ }_{A} k$ is a connected reductive group over the finite field $k$. Any such $k$-group is quasisplit ([35, Chapter III, $\S 2.2$, Theorem 1]). Thus $Z(k) \neq \emptyset$, hence $Z(A) \neq \varnothing$ by Hensel's lemma. There thus exists a Borel $A$-subgroup $B \subset G$. Let $T \subset B$ be its maximal $A$-torus. Over $K$, the $K$-group $G_{K}=G \times_{A} K$ contains the open set $U^{+} \times_{K} U^{-} \times_{K}\left(T \times_{A} K\right)$, where $U^{+} \subset B_{K}$ is the unipotent radical of $B_{K}$ and $U^{-}$is the unipotent radical of the opposite $K$-Borel subgroup of $B_{K} \subset G_{K}$. Each of these unipotent radicals is $K$-isomorphic to an affine space over $K$.

Let the $k$-torus $T_{0}=T \times_{A} k$ be split by a Galois field extension $k^{\prime} / k$. There exists an exact sequence of $k$-tori split by $k^{\prime} / k$

$$
1 \rightarrow Q_{0} \rightarrow P_{0} \rightarrow T_{0} \rightarrow 1,
$$

where $P_{0}$ is a quasitrivial $k$-torus and $Q_{0}$ is a flasque $k$-torus (Endo and Miyata, Voskresenskiŭ, cf. $\$ 1$ and $\S 5$ in [7], §0 in [9]).

Because $k$ is a finite field, the field extension $k^{\prime}$ of $k$ is cyclic. By a theorem of Endo and Miyata (see [7, Proof of Corollary 3, p. 200]), for any flasque $k$-torus $Q_{0}$ split by a cyclic extension $k^{\prime}$ of $k$, there exists a $k$-torus $Q_{1}$ split by $k^{\prime}$ such that $Q_{0} \times_{k} Q_{1}$ is $k$-isomorphic to a quasitrivial $k$-torus. If we let $K^{\prime} / K$ be the cyclic, unramified extension corresponding to $k^{\prime} / k$, and we let $A^{\prime} / A$ be the finite, connected, étale Galois cover given by the integral closure of $A \subset K$ in $K^{\prime}$, the sequence of characters associated to the above exact sequence enables us to produce a sequence of $A$-tori split by $A^{\prime} / A$,

$$
1 \rightarrow Q \rightarrow P \rightarrow T \rightarrow 1
$$


hence in particular a sequence of $K$-tori split by $K^{\prime} / K$

$$
1 \rightarrow Q_{K} \rightarrow P_{K} \rightarrow T_{K} \rightarrow 1,
$$

with $Q_{K}$ a direct factor of a quasitrivial $K$-torus and $P_{K}$ a quasitrivial $K$-torus (for basic facts on tori over arbitrary bases, including quasitrivial and flasque tori, see [9], $\S 0$ and $\S 1)$.

Because $Q_{K}$ is direct factor of a quasitrivial $K$-torus, Hilbert's theorem 90 implies that the projection $P_{K} \rightarrow T_{K}$ has a rational section, hence $Q_{K} \times_{K} T_{K}$ is $K$-birational to $P_{K}$, which is a $K$-rational variety. Now the product $G_{K} \times_{K} Q_{K}$ is $K$-birational to $U^{+} \times_{K} U^{-} \times_{K} P_{K}$, which is a $K$-rational variety.

Theorem 4.6. Let $A$ be the ring of integers of a $p$-adic field $K$, let $k$ be its residue field. Let $\mathcal{X} / A$ be a projective, flat curve over $A$. Assume that $\mathcal{X}$ is connected and regular, and that the (reduced) components of the special fibre are regular. Let $F$ be the function field of $\mathcal{X}$. Let $G / A$ be a (fibrewise connected) reductive group.

If a class in $H^{1}(F, G)$ is unramified at points of codimension 1 on $\mathcal{X}$, then it is trivial.

Proof. By Theorem 4.2 (i), there exists $\xi \in H^{1}(X, G)$ which restricts to the given class in $H^{1}(F, G)$. By hypothesis, each component $Y$ of the special fibre is a regular, hence smooth, projective curve over the finite field $k$. Let us show that the hypothesis of Theorem 4.2 (ii) is fulfilled. It is enough to show that for a smooth, projective, connected curve $Y / k$ and $G_{k}$ a connected reductive group the image of $H^{1}\left(Y, G_{k}\right)$ in $H^{1}\left(k(Y), G_{k}\right)$ is trivial. There exists a central extension of algebraic $k$-groups

$$
1 \rightarrow Q \rightarrow G_{k}^{\mathrm{sc}} \times P \rightarrow G_{k} \rightarrow 1
$$

where $G_{k}^{\text {sc }}$ is a simply connected semisimple $k$-group, $P$ is a quasitrivial $k$-torus and $Q$ is a flasque $k$-torus ([5, Proposition 3.1]). As recalled in the proof of Lemma 4.5, because $k$ is finite there exists a $k$-torus $Q_{1}$ such that $Q \times_{k} Q_{1}$ is a quasitrivial $k$-torus. The Brauer group $H^{2}\left(Y, \mathbb{G}_{m}\right)$ of a smooth projective curve $Y$ over a finite field is zero. Since this holds over any finite extension of $k$, this implies $H^{2}(Y, T)=0$ for any quasitrivial $k$-torus, hence for any $k$-torus $T$ which is a direct factor of a quasitrivial $k$-torus. Thus $H^{2}(Y, Q)=0$.

In the commutative diagram of exact sequences of pointed étale cohomology sets

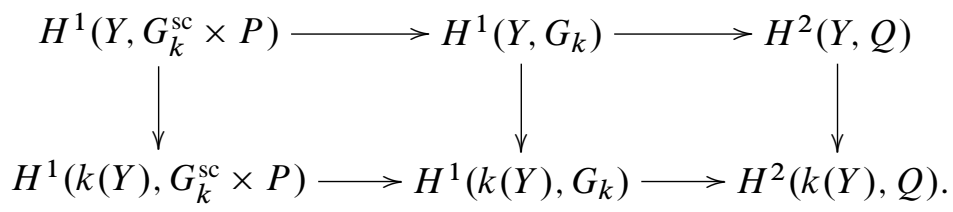

we have $H^{1}\left(k(Y), G_{k}^{\mathrm{sc}}\right)=1$ (Harder [17], [18]) and $H^{1}(k(Y), P)=0$ (Hilbert's theorem 90), and we have proved $H^{2}(Y, Q)=0$. Thus the image of $H^{1}\left(Y, G_{k}\right)$ in $H^{1}\left(k(Y), G_{k}\right)$ is trivial. 
The claimed result now follows from Lemma 4.5 and Theorem 4.2 (iii).

Remark 4.7. Applying Theorem 4.6 to the projective linear groups $\mathrm{PGL}_{n}$, one recovers a proof of Grothendieck's theorem that the Brauer group of a regular proper model $\mathcal{X} / A$ is trivial. That in its turn is closely related to the statement that an element of the Brauer group of $X$ which vanishes at each closed point of $X$ is trivial (Lichtenbaum [25]).

Theorem 4.8. Let $A$ be the ring of integers of a $p$-adic field $K$, let $k$ be its residue field. Let $X / K$ be a smooth, projective, geometrically integral curve. Let $F$ be the function field of $X$. Let $\Omega$ be the set of all discrete valuations on $F$. Let $G / A$ be a (fibrewise connected) reductive group. The restriction map with respect to completions $H^{1}(F, G) \rightarrow \prod_{v \in \Omega} H^{1}\left(F_{v}, G\right)$ has a trivial kernel.

Proof. One knows that $X / K$ admits a model $\mathcal{X} / A$ as in Theorem 4.6. Suppose that $\xi \in H^{1}(F, G)$ is in the kernel of the above restriction map. By Lemma 4.1, the class $\xi$ is unramified at points of codimension 1 on $\mathcal{X}$. We conclude by an application of Theorem 4.6.

Remark 4.9. For any integer $n$ and the $A$-group $G=\mathrm{PGL}_{n}$, in the above theorem one may replace $\Omega$ by the set $\Omega_{F / K}$ of discrete valuations on $F$ which are trivial on $K$ : this is just a reinterpretation of Lichtenbaum's theorem [25]. That this is not so for arbitrary $G$ is shown by the following example.

Let $p$ be an odd prime and $K=\mathbb{Q}_{p}$. Let $u$ be a unit in $\mathbb{Q}_{p}$ which is not a square. Let $X / K$ be the elliptic curve $y^{2}=x(x+1)(x-p)$. Let $F=K(X)$. For $a \in F^{*}$, let $(a) \in F^{*} / F^{* 2}=H^{1}(F, \mathbb{Z} / 2)$. Since the divisor of $x \in F^{*}$ on $X$ is divisible by 2 , the cup-product $\alpha=(x) \cup(u) \cup(p) \in H^{3}(F, \mathbb{Z} / 2)$ is unramified at places $v$ of $F$ trivial on $K$, hence is trivial in the completion $F_{v}$ at such a place. The prime $p$ defines a place on $F$, the residue field is the function field $\mathbb{F}_{p}(Y)$, where $Y$ is the curve defined by $y^{2}=x^{2}(x+1)$ over $\mathbb{F}_{p}$, which is birational to the curve $z^{2}=x+1$. The residue of $\alpha$ at that place is $\left(z^{2}-1\right) \cup(u) \in H^{2}\left(\mathbb{F}_{p}(Y), \mathbb{Z} / 2\right)$, and this class is nonzero, since it has a nontrivial residue at $z=1$.

This implies: for $G$ the split group of type $\mathrm{G}_{2}$, the restriction map

$$
H^{1}(F, G) \rightarrow \prod_{v \in \Omega_{F / K}} H^{1}\left(F_{v}, G\right)
$$

has a nontrivial kernel.

Lichtenbaum's theorem also implies that for any central simple algebra over $D$ over $K$, and $G$ the $F$-group PGL $_{D}$, the above map has a trivial kernel. The above example shows that this is not so for the $K$-group $G=\mathrm{SL}_{D}$, where $D$ is the quaternion algebra $(u, p)$ over $K=\mathbb{Q}_{p}$. 
Remark 4.10. Let $A$ be the ring of integers of a $p$-adic field $K$. Let $G / A$ be a (connected) reductive group. Let $F=K(X)$ be the function field of a smooth geometrically integral curve over $K$. Let $\mathcal{X}$ be a regular model of $X$ over $A$. Assume that the fibres of $G \rightarrow \operatorname{Spec} A$ are simply connected (this is equivalent to the assumption that the $K$-group $G \times_{A} K$ is simply connected). Then for $\xi \in H^{1}(F, G)$ and $x$ a point of codimension 1 on $\mathcal{X}$, defining a valuation $v$ on $F$ with associated completion $F_{v}$, the conditions

(i) $\xi$ is unramified at $x$ (as in Theorem 4.6)

(ii) $\xi$ has trivial image in $H^{1}\left(F_{v}, G\right)$ (as in Theorem 4.3 (i) and Theorem 4.8) are equivalent.

Indeed, for any point $x$ of codimension 1 on a regular model $\mathcal{X}$, with complete local ring $\hat{A_{x}}$ and residue class field $\kappa(x)$, we have $H^{1}\left(\widehat{A_{x}}, G\right) \simeq H^{1}(\kappa(x), G)$ (Hensel's lemma) and $H^{1}(\kappa(x), G)=1$ whether $x$ lies on the generic fibre of $\mathcal{X} / A$ (Kneser, Bruhat-Tits) or $x$ is a generic point of a component of the special fibre of $X / A$ (Harder [17], [18]).

\section{Connection to Rost's invariant and a theorem of Kato}

For any simply connected, absolutely almost simple semisimple group $G$ over a field $F$ of characteristic zero, we have Rost's invariant (see [22, Chapter VII, Section 31]):

$$
R_{G}: H^{1}(F, G) \rightarrow H^{3}(F, \mathbb{Q} / \mathbb{Z}(2)) .
$$

In a number of cases, this map has a trivial kernel. Such is the case if $G=\operatorname{SL}(D)$ for $D / F$ a central simple algebra of squarefree index (Merkurjev-Suslin). Such is the case for quasisplit groups of type ${ }^{3,6} D_{4}$ ([22, 40.16], [4, Theorem 6.14]) or of type $E_{6}, E_{7}$ (Garibaldi [10, Theorem 0.1], see also [4, Theorem 6.1]). Such is the case for the split group $G_{2}$ ([36, Theorem 9]). Such is the case for the split group $F_{4}$ $([36, \S 9.4])$. It is not reasonable to hope for a positive answer for an arbitrary such $G$, as examples with $G=\operatorname{Spin}(q)$ show.

For fields of cohomological dimension at most 2, the triviality of the kernel of the Rost invariant $R_{G}$ is none other than Serre's conjecture II for $G$, which in this generality is still unknown for $G$ of type $E_{8}$.

Remark 5.1. For fields of cohomological dimension 3 and $G$ arbitrary, $R_{G}$ may have a nontrivial kernel, as shown by the following example due to Merkurjev, and which we publish with his kind permission. There exists a field $k$ of characteristic 0 and of cohomological dimension 2 over which there exist a central simple division algebra $A=H_{1} \otimes_{k} H_{2}$ with $H_{1}$ and $H_{2}$ quaternion algebras ([27, Theorem 4]). Let $F$ be either $k(t)$ or $k((t))$. Then $F$ has cohomological dimension 3. The reduced norm of $A$ is a homogeneous form of degree 4 without a zero over $k$. Thus $t^{2} \in F$ is not 
a reduced norm of $A \otimes_{k} F$. That is, the class of $t^{2}$ in $F^{*} / \operatorname{Nrd}\left(A_{F}^{*}\right)=H^{1}(F, G)$, with $G=S L_{1}(A)$, is nontrivial. Let $[A] \in H^{2}\left(k, \mu_{4}\right) \subset \operatorname{Br} k$ be the class of $A$. By [22, p. 437] (for more details, see [26, p. 138]), the Rost invariant $R_{G}$ sends $t^{2} \in H^{1}\left(F, G_{F}\right)$ to the cup-product $t^{2} \cup[A] \in H^{3}\left(F, \mu_{4}^{\otimes 2}\right) \subset H^{3}(F, \mathbb{Q} / \mathbb{Z}(2))$ (here $t^{2}$ is taken in $F^{*} / F^{* 4}=H^{1}\left(F, \mu_{4}\right)$ ). Since $2[A]=0 \in \mathrm{Br} k$, this cup-product is zero.

When $G$ is quasisplit, not of type $E_{8}$, the situation is much better. The following proposition is certainly known to specialists.

Proposition 5.2. Let $F$ be a field of characteristic not 2 and of 2-cohomological dimension at most 3. Let $q_{0}$ be a quadratic form over $F$ which is isotropic and of dimension at least 5. Let $G=\operatorname{Spin}\left(q_{0}\right)$. Then the kernel of the Rost map $H^{1}(F, G) \rightarrow H^{3}(F, \mathbb{Q} / \mathbb{Z}(2))$ is trivial.

Proof. Let

$$
1 \rightarrow \mu_{2} \rightarrow \operatorname{Spin}\left(q_{0}\right) \rightarrow \mathrm{SO}\left(q_{0}\right) \rightarrow 1
$$

be the central isogeny from the Spin group to the special orthogonal group. This gives rise to an exact sequence of pointed Galois cohomology sets

$$
\mathrm{SO}\left(q_{0}\right)(F) \stackrel{\delta_{0}}{\rightarrow} H^{1}\left(F, \mu_{2}\right) \stackrel{i}{\rightarrow} H^{1}\left(F, \operatorname{Spin}\left(q_{0}\right)\right) \stackrel{j}{\rightarrow} H^{1}\left(F, \operatorname{SO}\left(q_{0}\right)\right) .
$$

For $\xi \in H^{1}\left(F, \operatorname{Spin}\left(q_{0}\right)\right)$, the class $j(\xi)$ corresponds to a quadratic form $q_{1}$ having $\operatorname{dimension} \operatorname{dim}\left(q_{0}\right)=\operatorname{dim}\left(q_{1}\right)$, discriminant $\operatorname{disc}\left(q_{0}\right)=\operatorname{disc}\left(q_{1}\right)$ and Clifford invariant $c\left(q_{0}\right)=c\left(q_{1}\right)$. Then in the Witt group $W(F)$ the class $q_{1} \perp-q_{0}$ is contained in the third power $I^{3}(F)$ of the fundamental ideal and its Arason invariant $e_{3}\left(q_{1} \perp-q_{0}\right) \in H^{3}\left(F, \mu_{2}\right)$, which coincides with the Rost invariant of $\xi$ ([22, p. 437]), is zero. Now the hypothesis $\operatorname{cd}_{2}(F) \leq 3$ implies that $H^{4}\left(F, \mu_{2}\right)=0$, $I^{4}(F)=0$ and that $e_{3}: I^{3}(F) \rightarrow H^{3}\left(F, \mu_{2}\right)$ is an isomorphism ([28], [32], and [1, Corollary 4, Theorem 2]). The two forms $q_{0}$ and $q_{1}$ have the same dimension. By Witt simplification they are isomorphic. Thus $j(\xi)=1$, hence $\xi=i(\eta)$ for some $\eta \in H^{1}\left(F, \mu_{2}\right)$. As $q_{0}$ is isotropic, the connecting map $\delta_{0}: \operatorname{SO}\left(q_{0}\right) \rightarrow H^{1}\left(F, \mu_{2}\right)=$ $F^{*} / F^{* 2}$, which is the spinor map, is onto. Thus $\xi=1 \in H^{1}\left(F, \operatorname{Spin}\left(q_{0}\right)\right)$.

Theorem 5.3. Let $F$ be a field of characteristic zero and of cohomological dimension at most 3. Let $G / F$ be an absolutely almost simple, simply connected, quasisplit semisimple group. Assume that $G$ is not of type $E_{8}$. Then the kernel of the Rost map $H^{1}(F, G) \rightarrow H^{3}(F, \mathbb{Q} / \mathbb{Z}(2))$ is trivial.

Proof. The cases ${ }^{1} A_{n}$ and $C_{n}$ are trivial, since in these cases $H^{1}(F, G)=1$ over any field $F$. For quasisplit groups of type ${ }^{3,4} D_{4}, E_{6}, E_{7}, G_{2}$ and $F_{4}$ the kernel is trivial over any field $F$ of characteristic zero (see references above). 
Let $G$ be of type ${ }^{2} A_{n}$, quasisplit, $n \geq 2$. There is a quadratic extension $L / F$ and an $L / F$-hermitian form $h$ of dimension $n+1$ such that $G=\mathrm{SU}(h)$. Further $G$ quasisplit implies that $G$ is isotropic ([2, 20.6 (ii), p. 225]), which in turn implies that the hermitian form $h$ is isotropic ([2, 23.8, p. 264]). Let $V$ be the underlying space of $h$. Then the map $q: V \rightarrow F$ given by $q(v)=h(v, v)$ is a quadratic form of dimension $2 n+2$ over $F$ which is isotropic. Further there is a homomorphism $\alpha: \operatorname{SU}(h) \rightarrow \operatorname{Spin}(q)$ such that the composite map

$$
H^{1}\left(F, \operatorname{SU}(h) \rightarrow H^{1}\left(F, \operatorname{Spin}(q) \rightarrow H^{3}(F, \mathbb{Q} / \mathbb{Z}(2)),\right.\right.
$$

is the Rost invariant for $\mathrm{SU}(h)$ where the first map is induced by $\alpha$ and has trivial kernel, and the second one is the Rost invariant for $\operatorname{Spin}(q)([22,31.44$, p. 438]). The triviality of the kernel of the Rost invariant in this case follows from Proposition 5.2.

Let $G$ be of type $B_{n}, n \geq 2$, or ${ }^{1} D_{n}$ or ${ }^{2} D_{n}, n \geq 3$, which is quasisplit. Then $G$ is isomorphic to $\operatorname{Spin}(q)$ for some quadratic form $q$ over $F$ of dimension at least 5; further, $G$ quasisplit implies that $G$ is isotropic, which in turn implies that the quadratic form $q$ is isotropic $([2,23.4$, p. 256]). In this case the triviality of the kernel of the Rost invariant follows from Proposition 5.2.

This completes the proof of the triviality of the kernel of the Rost invariant for all quasisplit groups not of type $E_{8}$.

By combining Theorem 5.3 and a theorem of Kato, one gets a proof of Conjecture 2 of the introduction for quasisplit groups without $E_{8}$-factors. That proof is independent of the other sections of the present paper.

Theorem 5.4. Let $K$ be a $p$-adic field. Let $X / K$ be a smooth, projective, geometrically integral curve. Let $F=K(X)$ be the function field of $X$. Let $\Omega$ denote the set of discrete valuations on the field $F$. Given $v \in \Omega$ we let $F_{v}$ denote the completion of $F$ at $v$. Let $G / F$ be a quasisplit, simply connected, absolutely almost simple group without $E_{8}$ factor. Then the kernel of the diagonal map $H^{1}(F, G) \rightarrow \prod_{v \in \Omega} H^{1}\left(F_{v}, G\right)$ is trivial.

Proof. The field $F=K(X)$ is of cohomological dimension 3. The result immediately follows from the combination of Theorems 5.3 and a theorem of Kato [20]: For $X / K$ as in the statement of the theorem, the kernel of the diagonal restriction map

$$
H^{3}(F, \mathbb{Q} / \mathbb{Z}(2)) \rightarrow \prod_{v \in \Omega} H^{3}\left(F_{v}, \mathbb{Q} / \mathbb{Z}(2)\right)
$$

is trivial (here it is enough to consider the $v$ 's associated to the codimension 1 points on a regular proper model of $X$ over the ring of integers of $K$ ).

The hypotheses of the above theorem should be compared with those of Theorem 4.8, whose proof builds upon the work of Harbater, Hartmann and Krashen. 
Using Theorem 4.8 together with Bruhat-Tits theory, we now show that Theorem 5.3 also holds for groups of type $E_{8}$ over $F(X)$.

Theorem 5.5. Let $A$ be the ring of integers of a $p$-adic field $K$. Let $X / K$ be a smooth, projective, geometrically integral curve. Let $F=K(X)$ be the function field of $X$. Let $G$ be an absolutely almost simple, simply connected semisimple group over $A$. If $G$ is of type $E_{8}$, assume that the residue characteristic is different from 2, 3 and 5. Then the kernel of the Rost map $H^{1}(F, G) \rightarrow H^{3}(F, \mathbb{Q} / \mathbb{Z}(2))$ is trivial.

Proof. As explained in the proof of Lemma 4.5, the group $G / A$ is automatically quasisplit.

We may restrict the set of places under consideration to the set of points of codimension 1 on a regular proper model $X / A$. Let $O_{v}$ be the ring of integers in $F_{v}$. The residue field $\kappa=\kappa_{v}$ at such a place is either a $p$-adic field or a function field in one variable over a finite field.

Let $F_{v}^{\mathrm{nr}}$ be the maximal unramified extension of $F_{v}$. At a prime $l$ different from the residue characteristic, the $l$-cohomological dimension of $F_{v}^{\text {nr }}$ is 1 , at the residue characteristic it is at most 2 ([35, Chapter II, §4.3, Proposition 12, p. 95]).

From this we deduce $H^{1}\left(F_{v}^{\mathrm{nr}}, G\right)=1$ if the residue characteristic is not a torsion prime of $G$; this would hold even if the connected group $G$ was not simply connected, as follows from Steinberg's theorem, see [36, Theorem 4"].

In the general case, i.e. when the residue characteristic is a torsion prime and the quasisplit group $G$ is simply connected and not of type $E_{8}$, we resort to the known (case by case) theorem that for such a group over a field $L$ of characteristic zero and of cohomological dimension 2, we know $H^{1}(L, G)=1$ (Merkurjev and Suslin, Bayer-Fluckiger and Parimala, Gille, Chernousov, see [36] and [12]). Under our hypotheses, we thus have $H^{1}\left(F_{v}^{\mathrm{nr}} / F_{v}, G\right) \stackrel{\simeq}{\rightarrow} H^{1}\left(F_{v}, G\right)$.

By Theorem 4.8, to prove the theorem it is enough to show that for any $v$ as above the kernel of the map $H^{1}\left(F_{v}, G\right) \rightarrow H^{3}\left(F_{v}, \mathbb{Q} / \mathbb{Z}(2)\right)$ is trivial.

Let $\xi \in H^{1}\left(F_{v}^{\mathrm{nr}} / F_{v}, G\right)$. Since $G / A$ is a reductive $A$-group, Bruhat-Tits theory, as developed in Gille's paper [11, Theorem $3^{\prime}$, Theorem $4^{\prime}$ ] shows that there exist a parahoric subgroup $P$ over $O_{v}$ and a class $\eta \in H^{1}\left(O_{v}, P\right)$ with the following properties.

The image of $\eta$ under the natural map $H^{1}\left(O_{v}, P\right) \rightarrow H^{1}\left(F_{v}^{\mathrm{nr}} / F_{v}, G\right)$ is $\xi$.

The reductive quotient $M / \kappa$ of $P \times O_{v} \kappa$ is part of an exact sequence of reductive groups over the field $\kappa$ :

$$
1 \rightarrow \mathbb{G}_{m} \rightarrow M^{\prime} \rightarrow M \rightarrow 1
$$

where $M^{\prime} / \kappa$ is the product of a simply connected semisimple group and a torus which is a direct factor of a quasitrivial torus. 
Let us consider the composite map

$$
\begin{array}{r}
H^{1}\left(F_{v}^{\mathrm{nr}} / F_{v}, G\right) \rightarrow \operatorname{Ker}\left[H^{3}\left(F_{v}, \mathbb{Q} / \mathbb{Z}(2)\right) \rightarrow H^{3}\left(F_{v}^{\mathrm{nr}}, \mathbb{Q} / \mathbb{Z}(2)\right)\right] \\
\rightarrow H^{2}(\kappa, \mathbb{Q} / \mathbb{Z}(1)) \stackrel{\stackrel{\sim}{\rightarrow}}{\rightarrow} \operatorname{Br}(\kappa),
\end{array}
$$

where the map from $H^{3}$ to $H^{2}$ is the usual residue map for primes different from the residue characteristic, and the Kato residue map in general. The image of $\xi$ under this composite map coincides with the image of $\eta$ under the composite map

$$
H^{1}\left(O_{v}, P\right) \stackrel{\simeq}{\rightarrow} H^{1}\left(\kappa, P \times O_{v} \kappa\right) \stackrel{\simeq}{\rightarrow} H^{1}(\kappa, M) \rightarrow H^{2}\left(\kappa, \mathbb{G}_{m}\right)=\operatorname{Br}(\kappa),
$$

where the map $H^{1}(\kappa, M) \rightarrow H^{2}\left(\kappa, \mathbb{G}_{m}\right)$ is the boundary map from the above sequence.

Since $\kappa$ is either a $p$-adic field or a function field in one variable over a finite field, $H^{1}\left(\kappa, M^{\prime}\right)=0$. Hence the map $H^{1}(\kappa, M) \rightarrow \operatorname{Br}(\kappa)$ has trivial kernel.

If the image of $\xi \in H^{1}\left(F_{v}, G\right)=H^{1}\left(F_{v}^{\mathrm{nr}} / F_{v}, G\right)$ in $H^{3}\left(F_{v}, \mathbb{Q} / \mathbb{Z}(2)\right)$ is zero, we conclude that $\eta=1$ hence that $\xi=1$.

\section{Appendix}

In this appendix we present a down-to-earth computation for the phenomenon mentioned in Remarks 3.3 and 4.4. Let $p$ be an odd prime. Let $E$ be the elliptic curve over $\mathbb{Q}_{p}$ defined by the affine equation

$$
y^{2}=x(1-x)(x-p) .
$$

Let $F=\mathbb{Q}_{p}(E)$ be its function field. We clearly have

$$
x+(1-x)=1 ; \quad x-(x-p)=p ; \quad(1-x)+(x-p)=1-p .
$$

Let $A \subset F$ be a proper discrete valuation ring, let $v$ denote the valuation, $A_{v}$ the completion of $A$ and $F_{v}$ the fraction field of $A_{v}$, i.e. the completion of $F$ at $v$. Let $k$ be the residue field of $A$. Let $\pi$ be uniformizing parameter for $A$. If $v(p)=0$, the field $k$ is a finite extension of $\mathbb{Q}_{p}$. If $v(p) \neq 0$ then $v$ induces on $\mathbb{Q}_{p}$ a proper valuation and $v(p)>0$. In both cases, $1-p$ is a square in $k$ hence is a unit which is a square in $A_{v}$.

Claim. The function $1-x$ is not a square in $F$, but it is a square in each completion $F_{v}$.

That $(1-x)$ is not a square is proved by considering the quadratic extension $F / \mathbb{Q}_{p}(x)$ : the kernel of the restriction map on square classes is $\mathbb{Z} / 2$, spanned by the class of $x(1-x)(x-p)$.

Assume $v(1-x)<0$. Then $v(x)=v(1-x)=v(x-p)$ hence (A1) gives that each of these is even. Let $x=u / \pi^{2 n}$ with $u \in A^{*}$ and $n>0$. From (A1) we get 
that $-u^{3}$, hence $-u$ is a square in $A_{v}$. Now $1-x=\left(\pi^{2 n}-u\right) / \pi^{2 n}$ is a square in $K_{v}$. Assume $v(1-x)>0$. Then $v(x)=0$ and $v(x-p)=0$. From (A1) we get $1-x=u \pi^{2 n}$ with $u$ a unit and $n>0$. Then from (A2) we get that $x$ and $x-p$ are squares in $A_{v}$. But then (A1) shows that $1-x$ is a square in $K_{v}$. Now assume $v(1-x)=0$. If $v(x)>0$ or $v(x-p)>0$, then (A2) implies that $1-x$ is a square in $A_{v}$. We are reduced to the case where $v(1-x)=v(x)=v(x-p)=0$. If $v(p)>0$, then $x(x-p)$ is a square in $A_{v}$. From (A1) we deduce that $1-x$ is a square in $A_{v}$.

We are reduced to the case $v(1-x)=v(x)=v(x-p)=0$, hence $v(y)=0$, and $v(p)=0$. That is, the valuation $v$ corresponds to a closed point $M$ on the elliptic curve $E$ over $\mathbb{Q}_{p}$ (the trivial valuation is excluded). The ring $A$ is the local ring of $E$ at that point. The point $M$ lies on the affine curve $y^{2}=x(1-x)(x-p)$. Let $k / \mathbb{Q}_{p}$ be the corresponding finite field extension. Thus $k$ is the residue field of $A_{v}$. Let $B$ be the ring of integers of $k$. The reductions of $x$ and $y$ modulo the maximal ideal of $A$ give rise to elements $a, b \in k$ with $b(1-b)(b-p) \neq 0$ and $a^{2}=b(1-b)(b-p) \neq 0$. The element $1-x$ is a square in $A_{v}$ if and only if $1-b$ is a square in $k$. To show that this is indeed the case, we do exactly the same computations in $k$, with respect to the valuation $w$ of $k$, which satisfies $w(p)>0$, as we had done in $F$. The computation is identical, it stops at the analogue of the end of the previous paragraph.

Acknowledgements. We thank D. Harbater for his remarks on the typescript.

Work for this paper was partially supported by Emory University (J.-L. ColliotThélène and V. Suresh), NSF grant DMS-0653382 (R. Parimala) and Max Planck Institut für Mathematik, Bonn (JLCT and RP).

\section{References}

[1] J. K. Arason, R. Elman and B. Jacob, Fields of cohomological 2-dimension three. Math. Ann. 274 (1986), 649-657. Zbl 0576.12025 MR 0848510

[2] A. Borel, Linear algebraic groups. Second, enlarged edition, Grad. Texts in Math. 126, Springer-Verlag, New York 1991. Zbl 0726.20030 MR 1102012

[3] A. Brumer, Remarques sur les couples de formes quadratiques. C. R. Acad. Sci. Paris Sér. $A-B 286$ (1978), no. 16, A679-A681. Zbl 0392.10021 MR 0498374

[4] V. Chernousov, The kernel of the Rost invariant, Serre's conjecture II and the Hasse principle for quasi-split groups ${ }^{3,6} D_{4}, E_{6}, E_{7}$. Math Ann. 326 (2003), no. 2, 297-330. Zbl 1036.20040 MR 1990912

[5] J.-L. Colliot-Thélène, Résolutions flasques des groupes linéaires connexes. J. reine angew. Math. 618 (2008), 77-133. Zbl 1158.14021 MR 2404747

[6] J.-L. Colliot-Thélène, Fibres spéciales des hypersurfaces de petit degré. C. R. Acad. Sc. Paris 346 (2008), 63-65. Zbl 1129.14059 MR 2385056 
[7] J.-L. Colliot-Thélène et J.-J. Sansuc, La R-équivalence sur les tores. Ann. Sci. École Norm. Sup. (4) 10 (1977), 175-229. Zbl 0356.14007 MR 0450280

[8] J.-L. Colliot-Thélène et J.-J. Sansuc, Fibrés quadratiques et composantes connexes réelles. Math. Ann. 244 (1979), no. 2, 105-134. Zbl 0418.14016 MR 0550842

[9] J.-L. Colliot-Thélène et J.-J. Sansuc, Principal homogeneous spaces under flasque tori: applications. J. Algebra 106 (1987), 148-205. Zbl 0597.14014 MR 0878473

[10] R. S. Garibaldi, The Rost invariant has trivial kernel for quasi-split groups of low rank. Comment. Math. Helv. 76 (2001), no. 4, 684-711. Zbl 1001.20042 MR 1881703

[11] P. Gille, Invariants cohomologiques de Rost en caractéristique positive. K-Theory 21 (2000), 57-100. Zbl 0993.20031 MR 1802626

[12] P. Gille, Cohomologie galoisienne des groupes quasi-déployés sur des corps de dimension cohomologique $\leq$ 2. Compos. Math. 125 (2001), 283-325. Zbl 1017.11019 MR 1818983

[13] J. Giraud, Cohomologie non abélienne. Grundlehren Math. Wiss. 179, Springer-Verlag, Berlin 1971. Zbl 0226.14011 MR 0344253

[14] D. Harbater and J. Hartmann, Patching over fields. Israel J. Math. 176 (2010), 61-107. Zbl 05728342 MR 2653187

[15] D. Harbater, J. Hartmann and D. Krashen, Applications of patching to quadratic forms and central simple algebras. Invent. math. 178 (2009), 231-263. Zbl 05627032 MR 2545681

[16] G. Harder, Halbeinfache Gruppenschemata über Dedekindringen. Invent. math. 4 (1967), 165-191. Zbl 0158.39502 MR 0225785

[17] G. Harder, Chevalley groups over function fields and automorphic forms. Ann. of Math. (2) 100 (1974), 249-306. Zbl 0309.14041 MR 0563090

[18] G. Harder, Über die Galoiskohomologie halbeinfacher algebraischer Gruppen. III. Collection of articles dedicated to Helmut Hasse on his seventy-fifth birthday, III. J. reine angew. Math. 274/275 (1975), 125-138. Zbl 0317.14025 MR 0382469

[19] B. Jacob and J.-P. Tignol, appendix to D. J. Saltman, Division algebras over p-adic curves. J. Ramanujam Math. Soc. 12 (1997), no. 1, 25-47; correction, ibid. 13 (1998), no. 2, 125-129. Zbl 0902.16021 MR 1462850

[20] K. Kato, A Hasse principle for two-dimensional global fields. J. reine angew. Math. 366 (1986), 142-183. Zbl 0576.12012 MR 0833016

[21] K. Kato and T. Kuzumaki, The dimension of fields and algebraic $K$-theory. J. Number Theory 24 (1986), no. 2, 229-244. Zbl 0608.12029 MR 0863657

[22] M.-A. Knus, A. Merkurjev, M. Rost and J.-P. Tignol, The book of involutions. With a preface in French by J. Tits, Amer. Math. Soc. Colloq. Publ. 44, Amer. Math. Soc., Providence, RI, 1998. Zbl 0955.16001 MR 1632779

[23] T. Y. Lam, The algebraic theory of quadratic forms. Math. Lect. Note Ser., W. A. Benjamin, Inc., Reading, Mass., 1973. Zbl 0259.10019 MR 0396410

[24] S. Lichtenbaum, Curves over discrete valuation rings. Amer. J. Math. 25, no. 2 (1968), 380-405. Zbl 0194.22101 MR 0230724

[25] S. Lichtenbaum, Duality theorems for curves over $p$-adic fields. Invent. math. 7 (1969), 120-136. Zbl 0186.26402 MR 0242831 
[26] A. S. Merkurjev, Rost invariants of simply connected algebraic groups. With a section by Skip Garibaldi, In Cohomological invariants in Galois cohomology, Univ. Lecture Ser. 28, Amer. Math. Soc., Providence, RI, 2003, 101-158. Zbl 1159.12311 MR 1999383

[27] A. S. Merkurjev, Simple algebras and quadratic forms. Izv. Akad. Nauk SSSR Ser. Mat. 55 (1991), no. 1, 218-224; English transl. Math. USSR-Izv. 38 (1992), no. 1, 215-221. Zbl 0763.12003 MR 1130036

[28] A. S. Merkurjev and A. A. Suslin, Norm residue homomorphism of degree three. Izv. Akad. Nauk SSSR 54 (1990), 339-356; English transl. Math. USSR Izv. 36 (1991), 349-368. Zbl 0716.19002 MR 1062517

[29] Ye. A. Nisnevich, Espaces homogènes principaux rationnellement triviaux et arithmétique des schémas en groupes réductifs sur les anneaux de Dedekind. C. R. Acad. Sci. Paris Sér. I Math. 299 (1984), no. 1, 5-8. Zbl 0587.14033 MR 0756297

[30] R. Parimala and V. Suresh, Isotropy of quadratic forms over function fields in one variable over $p$-adic fields. Inst. Hautes Études Sci. Publ. Math. 88 (1998), 129-150. Zbl MR

[31] R. Parimala and V. Suresh, The $u$-invariant of the function fields of $p$-adic curves. Ann. of Math. (2) 172 (2010), no. 2, 1391-1405. Zbl 1208.11053 MR 2680494

[32] M. Rost, On Hilbert Satz 90 for $K_{3}$ for degree-two extensions. Preprint, Regensburg, 1986; http://www.mathematik.uni-bielefeld.de/ rost/K3-86.html.

[33] L. H. Rowen, A. S. Sivatski and J.-P. Tignol, Division algebras over rational function fields in one variable. In Algebra and number theory, Hindustan Book Agency, Delhi 2005, 158-180. Zbl 1089.16015 MR 2193351

[34] S. Saito, Class field theory for curves over local fields. J. Number Theory 21 (1985), no. 1, 44-80. Zbl 0599.14008 MR 0804915

[35] J-P. Serre, Cohomologie galoisienne. Lecture Notes in Math. 5, Springer-Verlag, Berlin 1964; révisée et complétée, 1994. Zbl 0812.12002 MR 1324577

[36] J-P. Serre, Cohomologie galoisienne: progrès et problèmes. Séminaire Bourbaki, Exposé 783 (Mars 1994), Astérisque 227 (1995). Zbl 0837.12003 MR 1321649

[37] I. R. Shafarevich, Lectures on minimal models and birational transformations of two dimensional schemes. Notes by C. P. Ramanujam, Tata Institute of Fundamental Research Lectures on Mathematics and Physics 37, Tata Institute of Fundamental Research, Bombay 1966. Zbl 0164.51704 MR 0217068

Received December 1, 2009

Jean-Louis Colliot-Thélène, C.N.R.S., UMR 8628, Mathématiques, Bâtiment 425,

Université Paris-Sud, 91405 Orsay, France

E-mail: jlct@math.u-psud.fr

Raman Parimala, Emory University, 400 Dowman Drive, Atlanta, Georgia 30322, U.S.A.

E-mail: parimala@mathcs.emory.edu

Venapally Suresh, Emory University, 400 Dowman Drive, Atlanta, Georgia 30322, U.S.A.

E-mail: suresh@mathcs.emory.edu 